\title{
Über die automatische Registrierung der Hämolyse durch Serumkomplement und Lysolecithin
}

\author{
H. G. Siedentopf, K. Lauenstein * und H. Fischer \\ Max-Planck-Institut für Immunbiologie, Freiburg/Br. \\ (Z. Naturforschg. 20 b, 569—574 [1965] ; eingegangen am 23. Januar 1965)
}

\begin{abstract}
An experimental set up is described in which the degree of optical density during lysis of sensitized sheep erythrocytes by complement $\left(C^{\prime}\right)$ and other hemolytic agents is followed photometrically and recorded automatically.

The time required for $50 \%$ reduction in optical density is inversely proportional to the serum dilution and thus can be taken for quantitative measurement of $\mathrm{C}^{\prime}$-activity. The linear relationship forms the basis for a quantitative comparison of the effect of various inhibitors. A definite advantage of the method is due to the ease with which kinetic studies of whole complement and the intermediate steps of its action can be performed.

Using this method the influence of variations of the quantity of red cells, of antibody concentration, and of temperature are reported.

To demonstrate the usefulness of the method a number of experiments are given, each of which is characteristic for a certain field of application. They include estimation of lytic activity of serum complement, comparison of different $\mathrm{C}^{\prime}$ inhibitors, action of $\mathrm{C}^{\prime}$ on tanned erythrocytes in the absence of antibody, estimation of lytic activity of lysolecithin (LL), hemolysis by LL as obtained by activation of $\mathrm{C}^{\prime}$ in serum and from erythrocytes lysed by $\mathrm{C}^{\prime}$, inhibition of $\mathrm{C}^{\prime}$ and of LL by albumin and by lecithin.
\end{abstract}

Menschliches und tierisches Serumkomplement $\left(C^{\prime}\right)$ wird allgemein mit Hilfe Antikörper-beladener Erythrocyten bestimmt. Die Einstellung des hämolytischen Systems ist ebenso wie die Messung und Berechnung von $\mathrm{C}^{\prime}$ in hämolytischen Einheiten $\left(\mathrm{C}^{\prime} \mathrm{H}_{50}\right)$ heute weitgehend standardisiert ${ }^{1}$.

Ähnlich wie bei der einfachen $C^{\prime}$-Bestimmung wird bei kinetischen Studien des Ablaufs der $C^{\prime}$ Lyse verfahren. Den einzelnen Ansätzen werden Proben in kurzen Zeitabständen entnommen, und die Reaktion zwischen Zellen und $\mathrm{C}^{\prime}$ in geeigneter Weise unterbrochen. Nach raschem Zentrifugieren werden dann die Hämolysegrade der Überstände photometrisch bestimmt. Diese sampling technique ist aufwendig und nur möglich, wenn entweder mit limitierten Komplementmengen oder bei suboptimaler Antikörperbeladung gearbeitet wird.

Wünschenswert ist es, die Kinetik der Hämolyse auch bei hohen $\mathrm{C}^{\prime}$-Konzentrationen und massiver Antikörperbeladung zu verfolgen. Hierfür bietet sich die fortlaufende Registrierung der Hämolyse in einem optischen Test an. Obwohl schon frühzeitig und für verschiedene Fragestellungen der Hämolyseablauf photoelektrisch gemessen wurde (Mellanbx,

\footnotetext{
* Institut für experimentelle Pathologie der Farbenfabriken Bayer AG, Wuppertal.

1 E. A. Kabat u. M. M. MaYer, Experimental immunochemistry. II. ed. Charles C. Thomas, Springfield 1961.
}

Ponder, $\mathrm{Parpart}^{2}$ ), hat die fortlaufende Registrierung unseres Wissens bisher keinen Eingang in die $\mathrm{C}^{\prime}$-Forschung gefunden.

Im folgenden wird eine Versuchsanordnung geschildert, die es erlaubt, die Kinetik der Hämolyse durch Komplement oder auch durch andere Hämolysine photometrisch $\mathrm{zu}$ verfolgen und $\mathrm{zu}$ messen. Einige Anwendungsmöglichkeiten der Methode werden durch Beispiele belegt.

Das Prinzip ist einfach: Eine geeignet verdünnte Zellsuspension schwächt durchfallendes Licht ab. Nach Zugabe von $C^{\prime}$ oder einem hämolytischen Agens steigt die Lichtdurchlässigkeit parallel mit der Lyse der Zellen an. Man mißt den Übergang von „deckfarben“ zu „lackfarben“ am besten, indem man den zeitlichen Abfall der Extinktion automatisch registriert.

\section{Material und Methoden}

\section{Photometer und Schreiber}

Wir benutzten ein Photometer „Vitatron UFD“ mit angeschlossenem Schreiber UR 100. Es wurde bei einer Spaltbreite von $0,4 \mathrm{~mm}$, einer Wellenlänge von $545 \mathrm{~m} \mu$ und einer Schichtdicke von $1 \mathrm{~cm}$ gearbeitet (s. auch Standard-Versuchsanordnung).

2 E. Ponder, Hemolysis and related phenomena. Grune und Stratton, New York 1948. 


\section{Hämolytisches System}

Hammelblut wurde in ACD bei $4{ }^{\circ} \mathrm{C}$ im Kühlschrank aufbewahrt. Vor Gebrauch wurden die Zellen (E) 5-mal mit Puffer gewaschen, photometrisch auf $1 \cdot 10^{9}$ Zellen/ml eingestellt und im Volumenverhältnis 1 : 2 mit der entsprechenden Antikörperverdünnung $30 \mathrm{~min}$ bei $37{ }^{\circ} \mathrm{C}$ inkubiert. Die sensibilisierten Zellen (EA) wurden im Eisbad aufbewahrt. Die genaue Herstellung der Arbeitsverdünnung wird bei der StandardVersuchsanordnung beschrieben.

\section{Komplement $\left(C^{\prime}\right)$}

Meerschweinchen-Sammelserum wurde in kleinen Portionen bei $-70^{\circ} \mathrm{C}$ aufbewahrt und vor Gebrauch durch Einstellen in Eiswasser aufgetaut. In allen Serumproben wurde in Parallelansätzen die Gesamt- $C^{\prime}$. Aktivität in $\mathrm{C}^{\prime} \mathrm{H}_{50}$-Einheiten nach dem üblichen Verfahren bestimmt ${ }^{1}$.

\section{Puffer}

Es wurde Veronalpuffer $\left(p_{\mathrm{H}} 7,3\right)$ mit Ca-Mg-Zusatz verwendet ${ }^{1}$.

\section{Reagenzien}

Antikörper (A): Ambozeptor Behringwerke Marburg, Titer 1 : 6000, Kontr.-Nr.: 3521.

Lysolecithin (LL): Nutritional Biochemicals Corp., Cleveland/Ohio.

Äthylendiamintetraacetat (EDTA) : $\mathrm{Na}_{2} \mathrm{H}_{2}$-EDTA (Titriplex III, Merck, Darmstadt) wurde mit 1-n. $\mathrm{NaOH}$ auf $p_{\mathrm{H}} 7,4$ eingestellt, so daß vorwiegend $\mathrm{Na}_{3} \mathrm{H}$-EDTA vorlag.

Salicylaldoxim : p. a. Merck, Darmstadt.

EPL (Essentielle Phospholipide) : Nattermann, Köln. Human-Gammaglobulin: Behringwerke, Marburg. Op.-Nr.: 223.

Rinderserum-Albumin: trocken, „reinst“: BehringWerke, Op.-Nr.: 23356.

Antihumanglobulin-Serum: Molter, Heidelberg, Kontr.Nr. : 5209

\section{St a nd a rd - Versuchsanord nu ng}

Die Lyse wird im durchfallenden Licht verfolgt. Die Zellsuspension (E oder EA) wird so eingestellt, daß sie gegen Wasser eine Extinktion von 0,8 ergibt. Das entspricht einer Verdünnung von etwa $1: 26$ der Ausgangssuspension von $5 \cdot 10^{8}$ Zellen $/ \mathrm{ml}$ und, wie mehrfache Auszählungen ergaben, $1,9 \cdot 10^{7}$ Zellen $/ \mathrm{ml}$ in der Arbeitssuspension. Die Messungen wurden in einem klimatisierten Raum bei $21{ }^{\circ} \mathrm{C}$ durchgeführt. Sämtliche zum Versuch erforderlichen Reagenzien befanden sich in einem Eisbad. Die Reihenfolge der Zugabe wurde strikt eingehalten.

In die 1-cm-Küvette wird zunächst $0,1 \mathrm{ml} \mathrm{C}$-haltiges Serum bzw. Serumverdünnung pipettiert. Dann werden $2,5 \mathrm{ml}$ der eiskalten Zellsuspension aus einer Vollpipette mit großer Öffnung zugegeben. Gleichzeitig wird der Papiervorschub in Gang gesetzt. Die Zugabe im Strahl bewirkt intensives Durchmischen des $\mathrm{C}^{\prime}$ mit den Zellen und markiert exakt den Start der Reaktion. Während des Einlaufens der Zellsuspension steigt die
Extinktion auf etwa 0,8 an, d. h. auf den Wert, welcher $0 \%$-Hämolyse bedeutet. Die Lyse beginnt nach einer von der eingesetzten $C^{\prime}$-Menge abhängigen Latenzzeit (lag phase), die auch bei sehr hohen $\mathrm{C}^{\prime}$-Mengen mindestens 60 Sek. dauert. Die vollständige Hämolyse (100\%-Lyse) ist bei einer Extinktion von ca. 0,2 erreicht. Eine Originalkurve ist in Abb. 1 wiedergegeben.

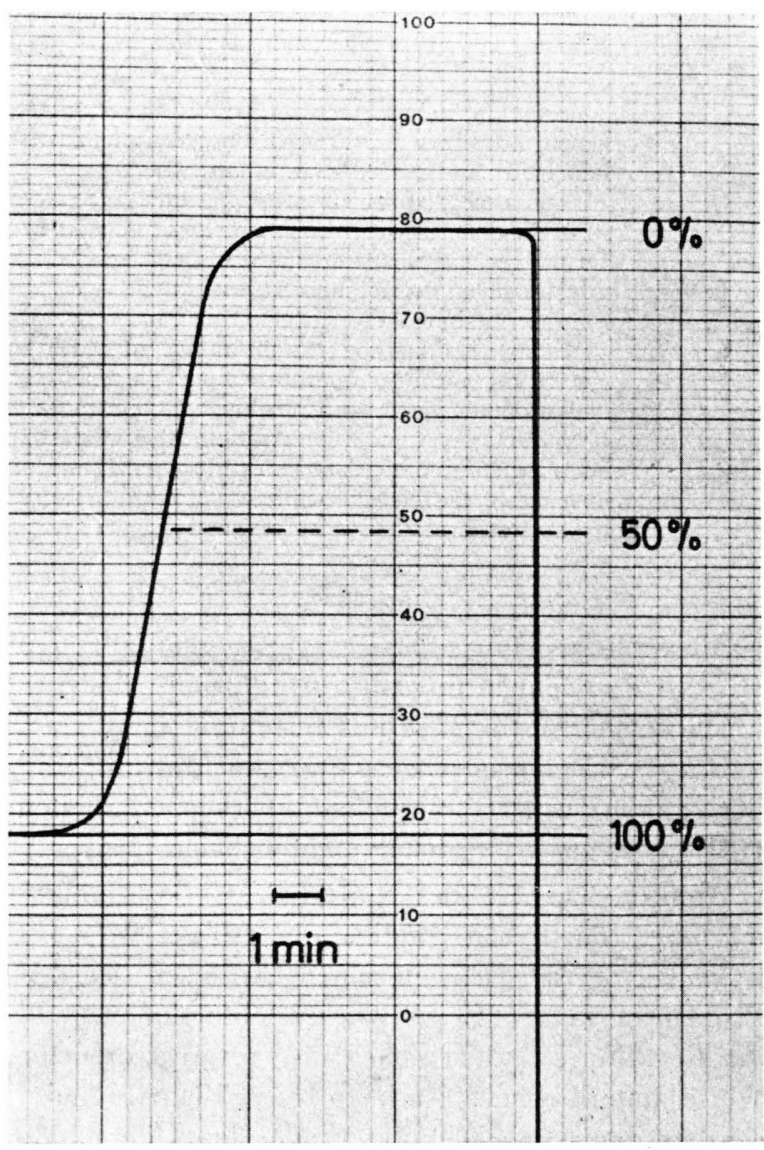

Abb. 1. Originalkurve der Lyse von $4,75 \cdot 10^{7}$ Zellen $/ 2,6 \mathrm{ml}$ durch $10 \mathrm{C}^{\prime} \mathrm{H}_{50}$ Komplement in 0,1 Milliliter. Alle weiteren Abbildungen sind Originalkurven, die durchgepaust und übereinandergezeichnet wurden.

Zur quantitativen Erfassung der Hämolyse benutzen wir die Zeit bis zur 50\%-Lyse. Der Wert der 50\%-Lyse läßt sich aus der erhaltenen Kurve leicht durch Halbieren der Differenz zwischen der Extinktion der 0\%- und der $100 \%$-Hämolyse bestimmen. Die bis zur 50\%-Lyse benötigte Zeit ist bei einem Papiervortrieb von $1 \mathrm{~cm} /$ Min. exakt abzulesen. An Stelle der 50\%-Hämolysezeit kann auch die planimetrisch gemessene Fläche des über der $50 \%$-Lyse liegenden Kurventeils benutzt werden. Wir fanden jedoch keine Unterschiede gegenüber der einfacheren Zeitmessung.

Um die Sedimentation der Zellen in der Küvette während des Meßvorgangs auszuschließen, haben wir in Vorversuchen die Stabilität der Erythrocyten-Suspen- 
sion geprüft. Dabei fand sich, da $\beta$ erst nach $30 \mathrm{Min}$. eine minimale Änderung der Extinktion durch Zellsedimentierung beobachtet wird. Dies gilt für Schaferythrocyten; Humanerythrocyten sedimentieren etwas schneller. Nur bei sehr langsam ablaufenden Reaktionen ist daher Rühren oder gelegentliches Durchmischen erforderlich.

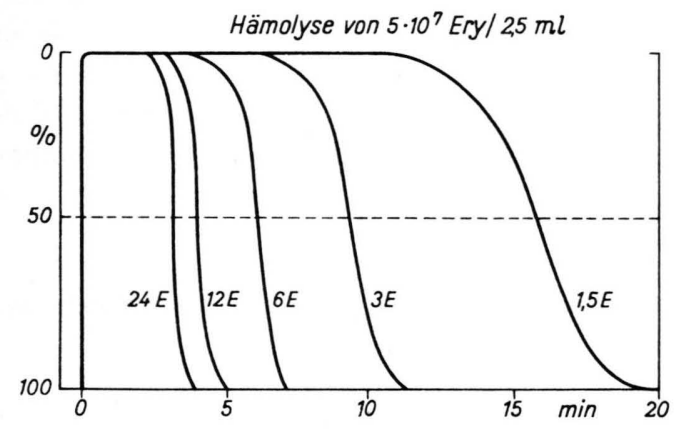

Abb. 2. Lysekurven bei Zimmertemperatur zur Aufstellung einer Eichkurve für $\mathrm{C}^{\prime}$.

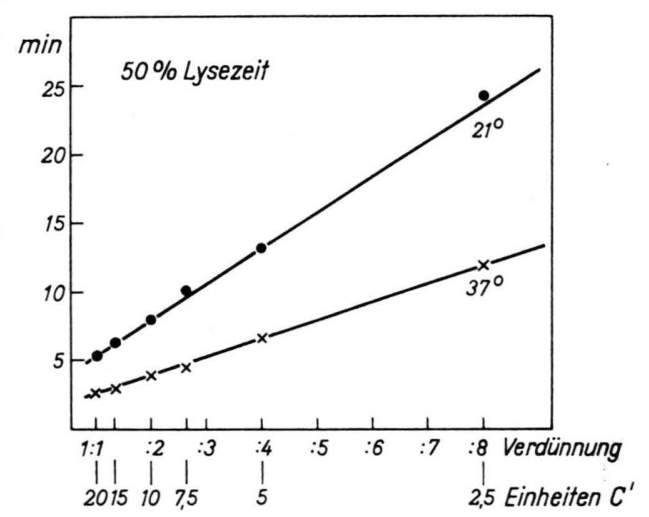

Abb. 3. Eichkurve für Komplement bei Zimmertemperatur $(\cdot-\cdot-\cdot)$ und bei $37^{\circ} \mathrm{C}(x-x-x)$.

Als Beispiel einiger Messungen ist in Abb. 2 und 3 die Auswertung der mit fallenden $C^{\prime}$-Mengen bei 2 verschiedenen Temperaturen erhaltenen Lysekurven dargestellt. Auf der Ordinate sind die 50\%-Lysezeiten und auf der Abszisse die $C^{\prime}$-Verdünnungen in arithmetischer Auftragung wiedergegeben. Die verwendete Serumverdünnung enthielt $240 \quad \mathrm{C}^{\prime} \mathrm{H}_{50} /$ Milliliter. Bei der gewählten Art der Auftragung erhält man Geraden, deren Neigungswinkel von der Reaktionstemperatur abhängig ist.

Wurde die Dichte der Erythrocyten-Suspension im geschilderten Originalansatz geändert, so zeigte sich, daß die Zellzahl bei konstanter Komplementmenge innerhalb eines relativ breiten Bereiches die Lysezeiten nicht beeinflußt (Tab. 1).

Um den Einfluß verschiedener Antikörperbeladung der Zellen zu ermitteln, prüften wir Antikörperverdünnungen von $1: 1400$ bis $1: 200$ (beginnende

\begin{tabular}{|c|c|c|c|}
\hline $\begin{array}{c}\text { Zellzahl } \\
\text { in } 2,5 \mathrm{ml} \\
{\left[\cdot 10^{7}\right]}\end{array}$ & \multicolumn{3}{|c|}{$\begin{array}{c}\text { 50\%-Lysezeit bei Einsatz von } \\
\text { [Min.] }\end{array}$} \\
& $5 \mathrm{C}^{\prime} \mathrm{H}_{50}$ & $10 \mathrm{C}^{\prime} \mathrm{H}_{50}$ & $20 \mathrm{C}^{\prime} \mathrm{H}_{50}$ \\
2,0 & 11,0 & 9,2 & 6,4 \\
4,0 & 11,5 & 9,0 & 6,2 \\
8,0 & 11,8 & 8,5 & 6,1 \\
16,0 & 13,3 & 8,9 & 6,1 \\
\hline
\end{tabular}

Tab. 1. Einfluß von Veränderungen der Zellzahl im Ansatz auf die $50 \%$-Lysezeiten bei verschiedenen $\mathrm{C}^{\prime}$-Mengen.

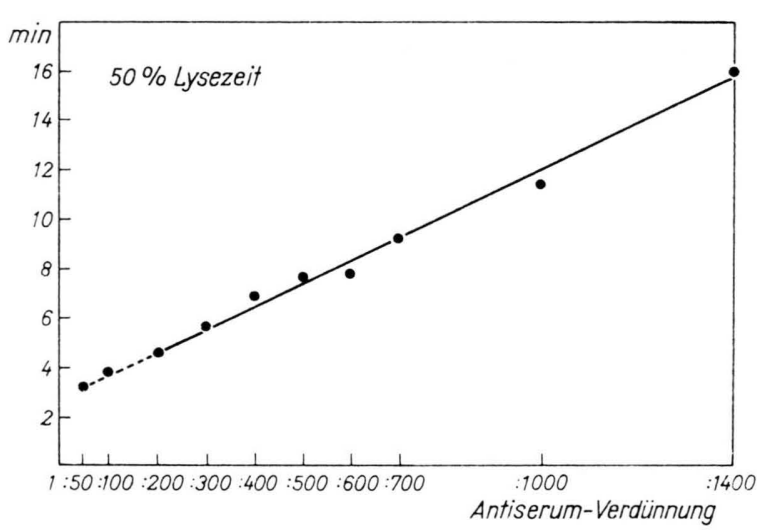

Abb. 4. Abhängigkeit der 50\%-Lysezeit von der Antikörperbeladung der Zellen.

Agglutination). Wie Abb. 4 zeigt, verhalten sich Antikörperverdünnung und Lysezeit über den gesamten Bereich proportional. $\mathrm{Ob}$ dies eine Eigenschaft des von uns verwendeten Antiserums ist oder durch das im $C^{\prime}$ Überschuß arbeitende System bedingt ist, muß zunächst noch offenbleiben (s. hierzu l. c. ${ }^{1}$, S. 146).

\section{Ergebnisse und Diskussion}

Die beschriebene Versuchsanordnung eignet sich zur quantitativen Bestimmung der Gesamtkomplement-Aktivität und zur Messung der Kinetik des Lyseablaufs; darüber hinaus ist sie auch für weitere Problemstellungen, bei denen eine Auflösung von Zellen erfolgt, zu verwenden. Dazu werden einige Beispiele gegeben:

\section{Vergleich von Hemmstoffen}

Die Verlängerung der 50\%-Lysezeit gibt ein reproduzierbares Maß für die Wirkung von $\mathrm{C}^{\prime}$-Hemmstoffen. In Abb. 5 ist die Hemmung der $\mathrm{C}^{\prime}$-Lyse durch EDTA, in Abb. 6 die durch Salicylaldoxim gezeigt. In der folgenden Arbeit ${ }^{3}$ werden Ergebnisse ausführlich beschrieben, die mit Hemmstoffen des Heparintyps gewonnen wurden. 


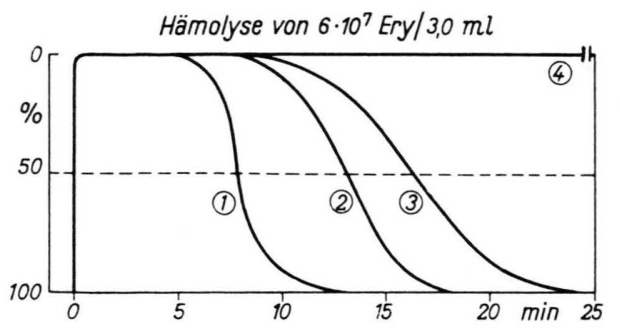

(1) $3,0 \mathrm{ml} \mathrm{EA}+20 \mathrm{EC}^{\prime} \mathrm{H}_{50}$, (2) $3,0 \mathrm{ml} \mathrm{EA}+20 \mathrm{EC}^{\prime} \mathrm{H}_{50}+$ EDTA (Endkonzentration $0,39 \mathrm{mMol}$ ), (3) $3,0 \mathrm{ml} \mathrm{EA}+$ $20 \mathrm{EC}^{\prime} \mathrm{H}_{50}+$ EDTA (Endkonzentration $0,42 \mathrm{mMol}$ ) ; (4) 3,0 $\mathrm{ml} \mathrm{EA}+20 \mathrm{EC}^{\prime} \mathrm{H}_{50}+\mathrm{EDTA}$ (Endkonzentration 0,6 mMol).

Abb. 5. Hemmwirkung von EDTA (Verdoppelung der 50\%. Lysezeit von $20 \mathrm{C}^{\prime} \mathrm{H}_{50}$ durch eine Konzentration von $0,4 \mathrm{mMol}$ ) .

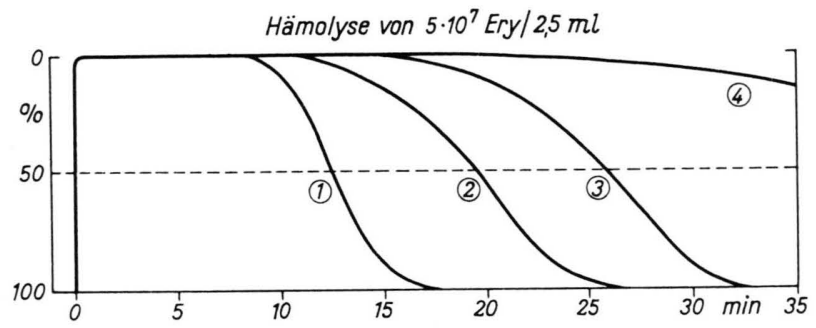

(1) $2,5 \mathrm{ml} \mathrm{EA}+\mathrm{C} 20 \mathrm{EC}^{\prime} \mathrm{H}_{50}$, (2) $2,5 \mathrm{ml} \mathrm{EA}+20 \mathrm{EC}^{\prime} \mathrm{H}_{50}+$ Salicylaldoxim (Endkonzentration $2,5 \mathrm{mMol}$ ), (3) $2,5 \mathrm{ml} \mathrm{EA}$ $+20 \mathrm{EC}^{\prime} \mathrm{H}_{50}+$ Salicylaldoxim (Endkonzentration 3,12 mMol), (4) $2,5 \mathrm{ml} \mathrm{EA}+20 \mathrm{EC}^{\prime} \mathrm{H}_{50}+$ Salicylaldoxim (Endkonzentration $3,75 \mathrm{mMol}$ ).

Abb. 6. Hemmwirkung von Salicylaldoxim (Verdoppelung der $50 \%$-Lysezeit von $20 \mathrm{C}^{\prime} \mathrm{H}_{50}$ durch eine Konzentration von $3,15 \mathrm{mMol}$ ) .

\section{Analyse der Hemmstoffwirkung}

Mit Hilfe von „persensibilisierten“ Zellen (KLEin) und den entsprechenden komplettierenden $\mathrm{C}^{\prime}$-Fraktionen ist es möglich, den Angriffsort von Hemmstoffen innerhalb der C'-Kette zu bestimmen. Wir haben von dieser Möglichkeit bei der Untersuchung verschiedener Heparinoide Gebrauch gemacht, über die auch in der folgenden Arbeit ${ }^{3}$ berichtet wird.

Aus der Form der Lysekurve persensibilisierter Zellen können Rückschlüsse auf die Aktivität des jeweiligen Systems gezogen werden. So ergeben mit $\mathrm{C}^{\prime}$-Faktoren gut beladene Zellen mit wenig aktivem R-Serum eine deutliche Verlängerung der Latenzzeit und eine anschließende langsame Lyse, während bei weniger beladenen Zellen und gutem R-Serum eine

${ }^{3}$ K. Lauenstein, H. G. Siedentopf u. H. Fischer, Z. Naturforschg. 20 b, 575 [1965].

4 S. Barandun, P. Kistler, F. Jeunet u. H. Isliker, Vox sanguinis 7, 157 [1962].

5 T. Ishizaka u. K. Ishizaka, Proc. Soc. exp. Biol. Med. 101, 845 [1959]. kürzere Latenzzeit mit anschließender sehr langsamer Lyse zu beobachten ist.

Um den Angriffspunkt in der Komplementkette zu bestimmen, versuchten wir zunächst, Hemmstoffe zu verschiedenen Zeitpunkten innerhalb der Latenzzeit zuzugeben. Dabei fand sich zwar eine Abhängigkeit der Hemmwirkung vom Zeitpunkt der Hemmstoffzugabe, jedoch konnten wir niemals eine Lyse nur eines Teiles der Zellen beobachten, wie dies von Mayer und Levine (cit. nach l.c. ${ }^{1}$, S. 174) bei der Zugabe von EDTA während der lag phase gefunden wurde. Auch dieses Ergebnis ist vermutlich auf den $\mathrm{C}^{\prime}$-Überschuß unseres Systems zurückzuführen.

\section{Komplementhämolyse ohne Antigen-Antikörper- Reaktion}

Aggregiertes humanes Gammaglobulin fixiert und aktiviert $\mathrm{C}^{\prime}$, einerlei, ob die Aggregation bei der Proteinreinigung ${ }^{4}$ durch Hitze ${ }^{5,6}$, Diazotierung ${ }^{7}$ oder Äthanol $^{8}$ bewirkt wird. Jeannet und Hässig ${ }^{9}$ diskutieren in diesem Zusammenhang, ob bestimmte Formen hämolytischer Anämien bei Makroglobulinämie durch Anlagerung und Aggregation dieser Makroglobuline an der Erythrocyten-Oberfläche verursacht werden. Auf Anregung von D. Rowley versuchten wir mit unserer Anordnung zu klären, in wieweit mit aggregiertem Gammaglobulin beladene Zellen für $\mathrm{C}^{\prime}$ anfällig sind.

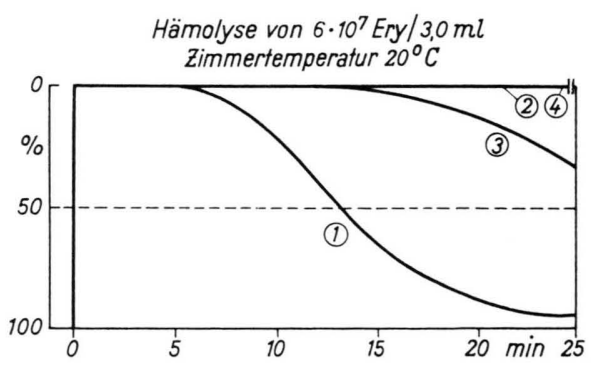

(1) $96 \mathrm{EC}^{\prime} \mathrm{H}_{50}+$ tannierte Erythrozyten + aggregiertes $\gamma$. Globulin $\left(5,3 \%\right.$, human) Coombstest ++++ , (2) $96 \mathrm{EC}^{\prime} \mathrm{H}_{50}$ + tannierte Erythrozyten $+\mathrm{NaCl}(0,9 \%)$ Coombstest $\phi$, (3) $96 \mathrm{EC}^{\prime} \mathrm{H}_{50}+$ normale Erythrozyten + aggregiertes $\gamma$-Globulin $\left(5,3 \%\right.$, human) Coombstest + , (4) $96 \mathrm{EC}^{\prime} \mathrm{H}_{50}+$ normale Erythrozyten $+\mathrm{NaCl}(0,9 \%)$ Coombstest $\varnothing$.

Abb. 7. C'-Lyse ohne Antigen-Antikörperreaktion (Näheres s. Text).

${ }^{6}$ B. D. Davis, E. A. Kabat, A. Harris u. D. H. Moore, J. Immunology 49, 223 [1944].

7 T. Ishizaka u. K. Ishizaka, J. Immunology 88, 690 [1962].

8 I. HaUPt, unveröffentlicht.

9 M. Jeannet u. A. Hässig, Vox sanguinis 9, 113 [1964]. 
Es wurden sowohl normale wie auch mit Tannin vorbehandelte Zellen mit 2,5-proz. aggregiertem humanen Gammaglobulin bei $p_{\mathrm{H}} 6,4$ inkubiert und anschließend mehrmals gewaschen. In beiden Fällen war Gammaglobulin angelagert worden, der Antihumanglobulin-Test ( $\mathrm{C}$ o o m b s) war bei den normalen Erythrocyten schwach und bei den tannierten stark positiv. Im Vergleich zu den entsprechenden Kontrollen zeigte die Prüfung in unserer Versuchsanordnung eindeutig, daß die $\mathrm{C}$ o o m b s - positiven Zellen durch $\mathrm{C}^{\prime}$ aufgelöst wurden. Bemerkt sei, daß es sich bei diesem Versuch um Erythrocyten aus einer 5 Wochen alten Konserve handelte. Bei frischen Zellen sind die Unterschiede erst nach längerer Inkubation deutlich (Abb. 7).

Das Versuchsbeispiel unterstreicht die seit langem bekannte, aber von Immunologen wenig beachtete Tatsache, daß auch ohne spezifischen Antikörper Zellen für den Angriff von $\mathrm{C}^{\prime}$ zugänglich sind. An anderer Stelle wird über die nicht-immunologische Komplementhämolyse gemeinsam mit $\mathrm{O}$. GörzE berichtet werden (s. auch Zit. 10).

\section{Hämolyse durch Lysolecithin}

(Versuche gemeinsam mit I. HAUPT)

Läßt man frisches Serum bei Zimmertemperatur oder bei $37^{\circ} \mathrm{C}$ altern, so entsteht vermehrt Lysolecithin, worauf bereits Bergenhem und FaHraeus

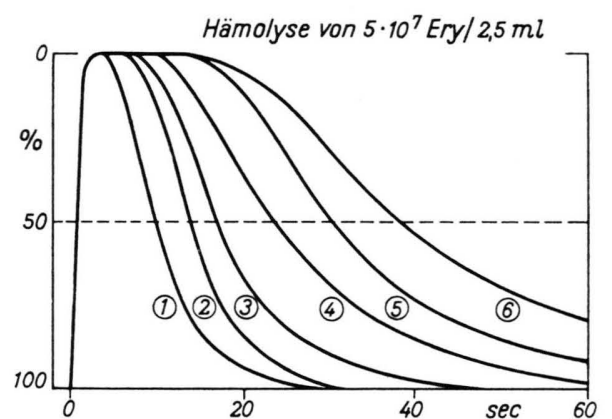

(1) $25 \gamma \mathrm{LL}$, (2) $12,5 \gamma \mathrm{LL}$, (3) $10 \gamma \mathrm{LL}$, (4) $7,5 \gamma \mathrm{LL}$, (5) $5,0 \gamma \mathrm{LL}$, (6) $3,75 \gamma \mathrm{LL}$.

Abb. 8. Lysekurven zur Aufstellung der Eichkurve für Lysolecithin bei Zimmertemperatur.

hingewiesen haben ${ }^{11}$. Das gleiche geschieht innerhalb weniger Min., wenn Komplement im Serum

10 H. Fischer u. I. Haupt, 15. Colloquium Ges. physiol. Chem. am 23./25. Apr. 1964, Mosbach/Baden, Springer-Verlag, Heidelberg, in Vorbereitung.

11 B. Bergenhem u. R. Fahraeus, Z. ges. exp. Med. 97, 555 [1936]. aktiviert wird ${ }^{12-15}$. Bei der offensichtlichen Verknüpfung zwischen vermehrter Lysolecithinbildung und $\mathrm{C}^{\prime}$-Aktivierung gewinnt der Nachweis sehr kleiner Lysolecithinmengen an Interesse. Er läßt sich mit

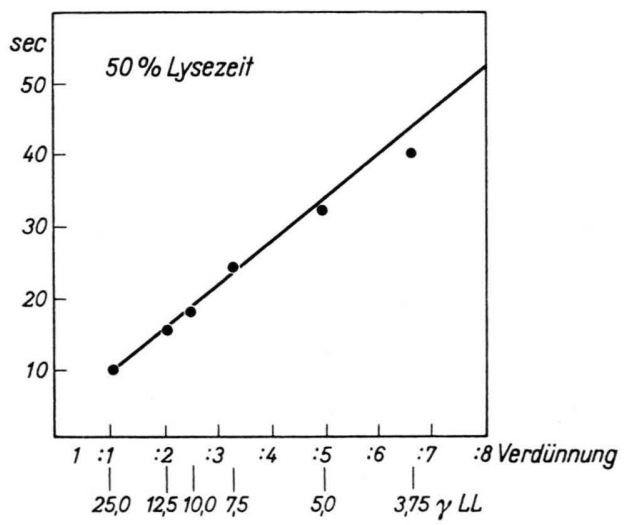

Abb. 9. Aus Abb. 8 gewonnene Eichkurve für Lysolecithin.

der beschriebenen Anordnung leicht durchführen. Wie Abb. 8 und 9 zeigen, kann die graphisch registrierte Hämolyse durch Lysolecithin ebenso für die quantitative Bestimmung herangezogen werden wie für die Messung von $\mathrm{C}^{\prime}$-Aktivitäten. Da die Lyse durch Lysolecithin sehr viel schneller abläuft als die $\mathrm{C}^{\prime}$-Lyse, muß man lediglich einen schnelleren Papiervorschub (15 cm/Min.) benutzen. Außerdem ist auf genaue Einhaltung der Zellzahl zu achten.

In Abb. 10 ist ein Beispiel für die Bestimmung des im Serum bei $C^{\prime}$-Aktivierung im Vergleich zur Kontrolle vermehrt gebildeten LL gegeben. Wie die linke Seite der Abb. zeigen soll, wurden die Lipid-

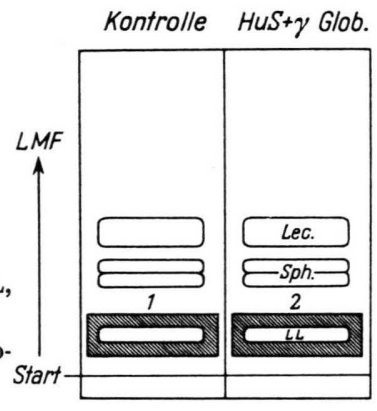

Abb. 10. Bildung von LL nach Zusatz von aggregiertem Gammaglobulin (Näheres s. Text).

12 H. Fischer u. I. Haupt, Naturwissenschaften 47, 137 [1960]. 13 H. Fischer u. I. HaUpt, Z. Naturforschg. 16 b, 321 [1961].

14 H. Fischer, Z. Immunitäts- u. Allergieforschung 126, 131 [1964].

15 H. Fischer, Ann. New York Acad. Sci. 116, 1063 [1964]. 
extrakte des normalen und des komplentaktivierten Serums durch Dünnschichtchromatographie auf Kieselgel getrennt und die LL-Zonen eluiert. Die lytische Aktivität der Eluate ist in den Lysekurven der rechten Seite der Abb. wiedergegeben. Die Abb. zeigt deutlich die erhöhte Lysolecithin-Aktivität im Komplement-aktivierten Serum.

Abb. 11 zeigt die Prüfung der aus Erythrocytenstroma mit der gleichen Methode extrahierten, getrennten und eluierten LL-Banden. In zahlreichen Versuchen ergab sich übereinstimmend, daß Stroma $\mathrm{C}^{\prime}$-gelöster Schaferythrocyten mehr LL enthält als

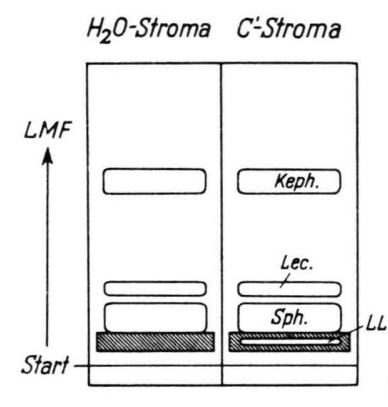
Hämolyse von $5 \cdot 10^{7} \mathrm{Ery} / 2,5 \mathrm{ml}$

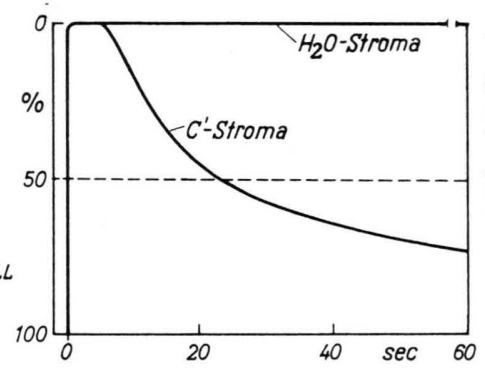

\section{Hemmung der Lysolecithinlyse durch Albumin}

Ohne Schwierigkeiten können im geschilderten Ansatz Hemmstoffe für LL geprüft werden. Ähnlich wie bei der Hemmung der Komplementlyse ist die Verlängerung der 50\%-Lysezeit ein gutes Maß zum Vergleich der Wirkung verschiedener Hemmstoffe. Wie Abb. 12 zeigt, hemmt Rinderalbumin bereits in sehr kleiner Konzentration die Lysolecithinlyse deutlich.

\section{Hemmung der Lysolecithinlyse und der Komplementlyse}

Die Suche nach Stoffen, die sowohl Komplement wie auch Lysolecithin hemmen, ist bei den oben erwähnten Zusammenhängen von besonderem Interesse. Wir fanden, daß Sojalecithin neben Albumin in dieser Hinsicht besonders wirksam ist und, wie Abb. 13 zeigt, zu einer deutlichen Hemmung beider Systeme führt ${ }^{17}$.

a Hämolyse von $5 \cdot 10^{7} \mathrm{Ery} / 2,5 \mathrm{ml}$

Abb. 11. Bildung von LL nach C'-Lyse (Näheres s. Text).

das Stroma entsprechender Kontrollen ohne C'Lyse ${ }^{10,16}$. Dieser wichtige Befund wird z. Z. weiter analysiert.

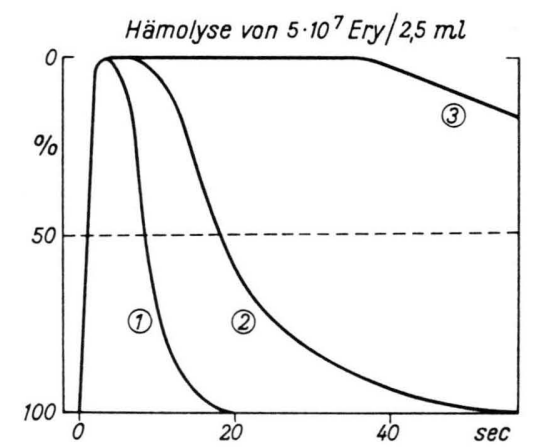

(1) $25 \gamma \mathrm{LL}$, (2) $25 \gamma \mathrm{LL}+500 \gamma$ Albumin (Endkonzentration ca. 0,02 g-\%), (3) $25 \gamma \mathrm{LL}+800 \gamma$ Albumin (Endkonzentration ca. $0,03 \mathrm{~g} . \%)$.

Abb. 12. Hemmwirkung von Albumin (Verdoppelung der $50 \%$-Lysezeit von $25 \gamma$ LL durch eine Konzentration von ca. $0,02 \mathrm{~g}-\%)$.

16 I. Haupt u. H. Fischer, Abstr. Xth Congr. int. Soc. Haematol. Stockholm 1964, K: 66.

17 H. G. Siedentopf, I. Haupt u. H. Fischer, Abstr. Xth Congr. int. Soc. Haemotol. Stockholm 1964, K: 64.

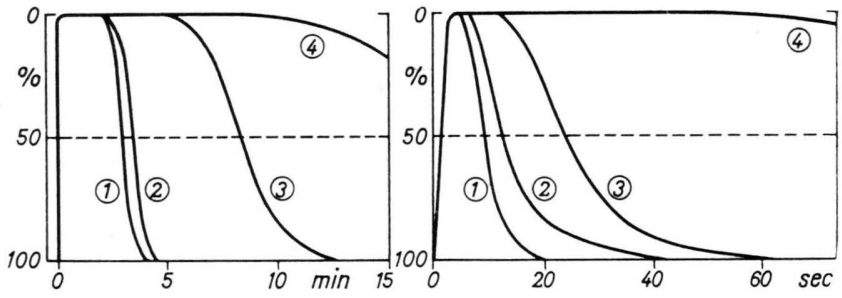

$\mathrm{Zu} \mathrm{a}$ : (1) $7,5 \mathrm{EC}^{\prime} \mathrm{H}_{50}$, (2) $7,5 \mathrm{EC}^{\prime} \mathrm{H}_{50}+400 \gamma \mathrm{EPL}$ (Endkonzentration ca. $0,016 \mathrm{~g}-\%)$, (3) $7,5 \mathrm{EC}^{\prime} \mathrm{H}_{50}+5 \mathrm{mg} \mathrm{EPL}$ (Endkonzentration ca. 0,2 g-\%), (4) $7,5 \mathrm{EC}^{\prime} \mathrm{H}_{50}+10 \mathrm{mg}$ EPL (Endkonzentration ca. 0,4 g-\%). - Zu b: (1) $25 \gamma$ LL,

(2) $25 \gamma \mathrm{LL}+25 \gamma \mathrm{EPL}$ (Endkonzentration ca. 0,001 g-\%),

(3) $25 \gamma \mathrm{LL}+50 \gamma \mathrm{EPL}$ (Endkonzentration ca. 0,002 g.\%),

(4) $25 \gamma \mathrm{LL}+100 \gamma \mathrm{EPL}$ (Endkonzentration ca. 0,004 g-\%). Abb. 13. Hemmwirkung von Sojalecithin auf Komplement und Lysolecithin (Verdoppelung der $50 \%$-Lysezeit von 7,5 $\mathrm{C}^{\prime} \mathrm{H}_{50}$ durch eine Konzentration von ca. 0,11 g-\% und von $25 \gamma \mathrm{LL}$ durch ca. $0,0015 \mathrm{~g}-\%)$.

Die fortlaufende optische Registrierung der Cytolyse, wie sie in der vorliegenden Arbeit speziell für die Lyse von roten Blutkörperchen durch Komplement und Lysolecithin beschrieben wurde, ist in dieser einfachen Anordnung auch zur Verfolgung der osmotischen Lyse von Erythrocyten anzuwenden. Sie ist aber nicht auf diese eine Zellart beschränkt. Orientierende Versuche haben gezeigt, daß auch die $\mathrm{C}^{\prime}$-induzierte Cytolyse von kernhaltigen Zellen und von Bakterien nach dem gleichen oder geringfügig abgeänderten Prinzip kinetisch analysiert werden kann. 